\title{
Tritium permeation experiment at IFMIF Medium Flux Test Module
}

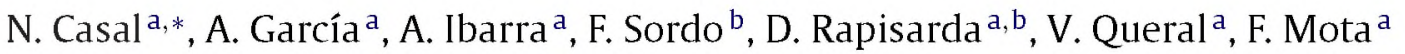 \\ a Laboratorio Nacional de Fusión, EURATOM-CIEMAT, 28040 Madrid, Spain \\ ${ }^{\mathrm{b}}$ Universidad Politécnica de Madrid, 28006 Madrid, Spain
}

Keywords:

IFMIF

DEMO

Liquid breeders

Tritium permeation

\begin{abstract}
A B S T R A C T
Tritium release experiments using different breeding material candidates are planned for the medium flux region of the IFMIF Test Cell. Nowadays, only ceramic breeder materials have been suggested to be tested in the Tritium Release Module located in the Medium Flux Test Module of IFMIF. Liquid breeder blankets are very promising and for that reason, several concepts will be tested in ITER. One of the main problems concerning the liquid blankets is the permeation of the generated tritium in the breeder throughout the walls. Since tritium permeation is highly influenced by irradiation conditions, IFMIF is a suitable scenario to perform tritium permeation related experiments.

In this paper, a preliminary design of a tritium permeation experiment for the Medium Flux Test Module of IFMIF is proposed, in order to contribute to the progress of the liquid breeder blanket concept validation.

The conceptual design of the capsule in which the experiment will be performed is carried out, taking into consideration the experiment necessities and its implementation in the Tritium Release Module. In addition to this, some thermal hydraulic calculations have been performed to evaluate the thermal behaviour of the irradiation capsule.
\end{abstract}

\section{Introduction}

IFMIF (International Fusion Materials Irradiation Facility) is an accelerator based neutron source aiming to test materials in a fusion reactor conditions. For this, high energy deuterons produced by two linear accelerators will impact in a target of flowing liquid lithium. The stripping reactions in the target will produce a neutron flux of adequate characteristics (neutron spectrum, He production/dpa ratio, PKA spectrum, etc.) for the study of materials of interest in the fusion field. IFMIF will be the unique specific tool for the qualification of materials capable of withstanding $14 \mathrm{MeV}$ neutrons being, like that, the essential next step towards the future reactor DEMO.

The high neutron flux region of IFMIF (50-20-dpa/fpy, 0.5 I) will accommodate the High Flux Test Module (HFTM) dedicated to test structural materials. In the medium neutron flux region (20$1 \mathrm{dpa} / \mathrm{fpy}, 6 \mathrm{l}$ ) the Medium Flux Test Module (MFTM) will be located. The MFTM will house, in its present design, two different experiments. First of all, and closer to the HFTM, the Creep Fatigue Test Module (CFTM), dedicated to in situ creep-fatigue experiments on structural materials, and the Tritium Release Module (TRM) dedicated to in situ tritium release experiments on ceramic breeders. Besides, these two sub-modules, two tungsten plates and a carbon envelope have been added to the MFTM in order to improve its neutronics performance for ceramic breeder materials [1].

\footnotetext{
* Corresponding author.

E-mail address: natalia.casal@ciemat.es (N.Casal).
}

For the time being, no validation experiments of the liquid breeder materials have been considered in the medium flux region. Since one of the major problems of the Helium-Cooled LithiumLead blanket (HCLL), one of the DEMO blanket candidate, is the tritium permeation throughout the structural material to the helium channels, a tritium permeation experiment through steels is proposed, being the aim of this paper to describe the conceptual design for such experiment.

\section{Liquid breeder blankets and tritium permeation R\&D}

During last years, liquid metal blankets have become a promising option since they allow a high operating temperature, an adequate tritium breeding ratio without beryllium neutron multiplier, easy maintenance and low pumping power. Nevertheless, several problems appear associated with liquid metals like magnetohydrodynamic (MHD) effects, tritium permeation and tritium control, liquid-structural material compatibility, etc., depending on the blanket design.

Some liquid blankets concepts have been developed in Europe, being among them, the Helium-Cooled Lithium-Lead (HCLL) blanket, based on limited extrapolation of current technology. This one is considered as possible DEMO blanket candidate, and it has been chosen for a TBM to be installed and tested in ITER.

The HCLL blanket is based on the use of EUROFER as structural material, $\mathrm{Pb}-17 \mathrm{Li}$ ( $\mathrm{Li}$ at $90 \%$ in $6 \mathrm{Li}$ ) as breeder, neutron multiplier and tritium carrier, and helium as coolant [2]. In each HCLL blan- 


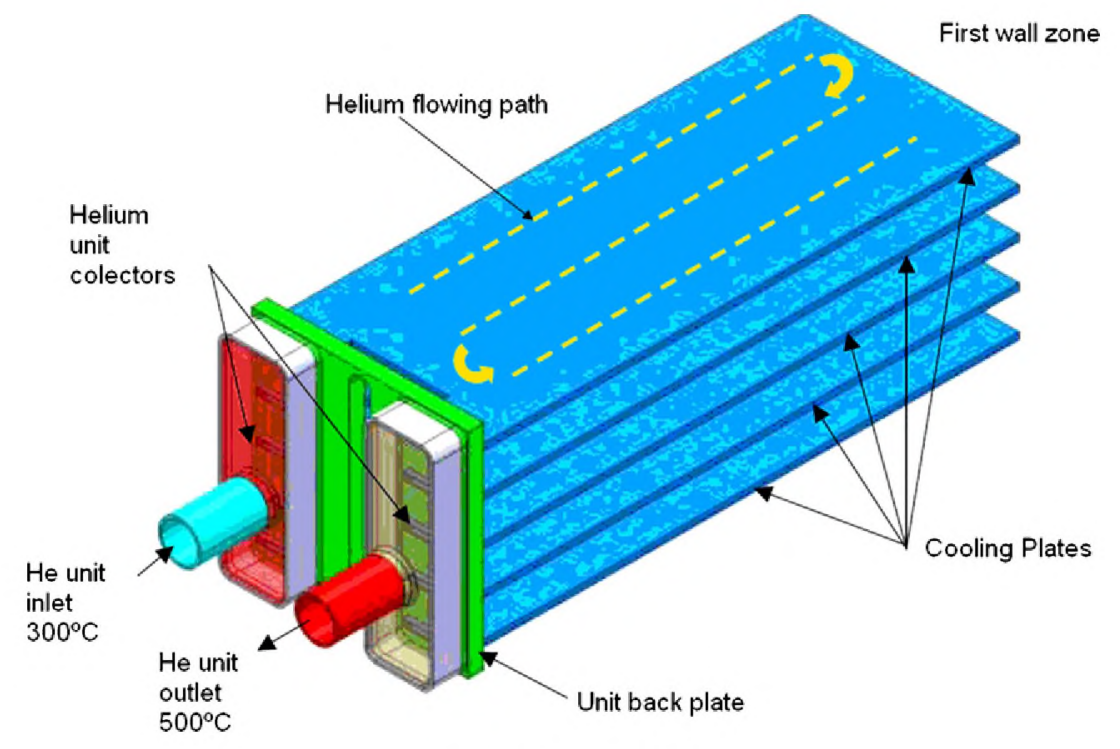

Fig. 1. HCLL breeder unit.

ket breeder unit (Fig. 1), the lithium lead (LiPb) flows between the cooling plates and the stiffening plates (plates which separate two adjacent breeder units) at low velocity, some $\mathrm{mm} / \mathrm{s}$. It is cooled by flowing helium flowing at around $40 \mathrm{~m} / \mathrm{s}$ inside the cooling plates, where maintain the range of temperatures from about $420^{\circ} \mathrm{C}$ to $490^{\circ} \mathrm{C}$ [3].

In the HCLL blanket, the tritium permeation will mainly occur through the cooling plates in a percentage of $84 \%$ over the total permeation (14.5\% across the stiffening plates, $1.4 \%$ across the first wall and $0.4 \%$ across the back plate) [3]. Furthermore, permeation is considered the most likely source of tritium leaks [4,5]. Some factors contribute to this high expected tritium permeation rate such as:

- A high tritium concentration in the channels owing to the low velocity in LiPb (used to prevent MHD effects).

- A high expected tritium partial pressure.

- A high diffusivity of tritium in EUROFER.

The use of permeation coatings to prevent a large amount of tritium in the helium coolant seems then to be required.

Although some experiments and studies have been performed during last years (i.e. [6-8]) in order to clarify the tritium management in a liquid breeder blanket, their results show a dramatic discrepancy between solubility of tritium [7], with the subsequent uncertainties in the estimated permeation rate towards the $\mathrm{He}$ coolant [4]. On the other hand, a large amount of experiments have been carried out without an irradiation environment (the antipermeation effect of these coatings is expected to be reduced in presence of irradiation due to the wall degradation).

Therefore, the study of the permeation coatings behaviour under irradiation is necessary, and in this line, the aim of this paper is to propose a tritium permeation experiment for the Medium Flux Test Module of IFMIF. The experiment will test the durability and antipermeation effect of permeation coatings to be used in DEMO liquid metal blankets.

\section{Conceptual design of a tritium permeation experiment under IFMIF scenario}

The conceptual design of the experiment has been performed considering as starting point the present design of the TRM of IFMIF already oriented to other experiments. So, work consists in the development of a rig, specially adapted for this module, in which LiPb is allocated aiming to generate tritium to be permeated throughout the walls and measure it subsequently. The proposed design is described in Section 3.2. In the section below, the suitability of the MFTM is discussed.

\subsection{Suitability of the medium flux region of IFMIF for testing liquid breeders}

As previously mentioned, the medium flux region of IFMIF was considered an adequate scenario to test tritium permeation in

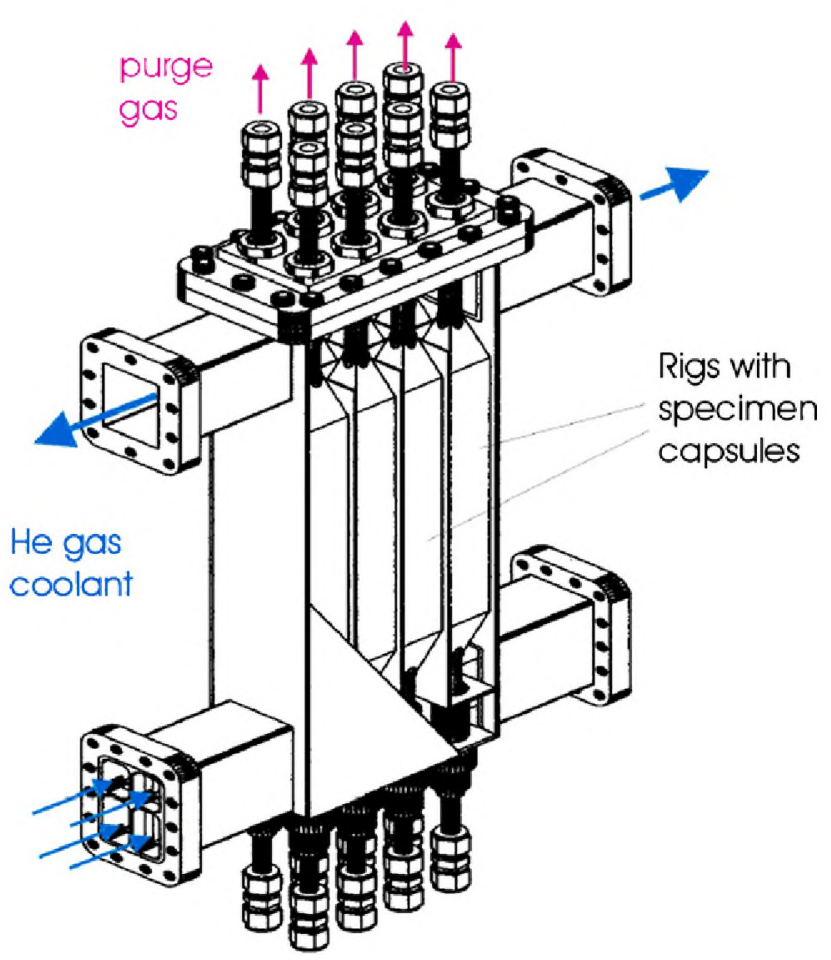

Fig. 2. Part of the TTC model for neutronic calculations (horizontal section). In the case represented here, the TRM is located behind the moderator and all the TRM rigs are considered filled with $\mathrm{LiPb}$. 
ceramic breeders. In that sense, a modification was introduced on the CDR (Comprehensive Design Report) design [9], in order to improve its neutronics performance for testing ceramic breeders, adding two tungsten plates and a carbon envelope as a new module inside the MFTM (Moderator Module, MM) [1].

To assess the suitability of this region also for testing liquid breeders, some draft neutronics calculations have been performed to compare the irradiation conditions of the medium flux region with the existing on a HCLL DEMO blanket.

These calculations have been carried out by using McDelicious code [10]. This code was developed by FZK (Karlsruhe, Germany) as an enhancement to MCNP5 code [11], in order to adapt computational requirements of IFMIF Test Cell, by simulating accurately the generation of D-Li source neutrons and introducing a threedimensional geometric model of the IFMIF Test Cell.

The calculations have been performed considering the Test Cell geometrical model from [12], but including some rigs filled with liquid $\mathrm{LiPb}$ in different positions of TRM, instead of ceramic breeder samples. Fig. 2 shows a partial view of the model for the neutronic calculations, showing the area of study.

The expected values of $\mathrm{dpa} / \mathrm{fpy}$ and the helium generation in the capsule wall have been calculated and compared with the representative values of a HCLL fusion power reactor blanket $[13,14,16]$. Also, tritium generated in the $\mathrm{LiPb}$ has been calculated and compared. Three different configurations of the MFTM have been considered for the calculations: TRM in the position of CFTM (directly behind the HFTM), TRM after the CFTM but with the MM installed and, finally, TRM after the CFTM and the MM removed. Results with all the rigs in the TRM filled with LiPb are shown in Table 1.

When the TRM is installed in its reference position (behind the CFTM), the expected damage and helium production in the walls of the capsule are in the range of expected values in the breeder zone of a HCLL fusion power reactor blanket (Table 1). Nevertheless the values are still low ( $2.6 \mathrm{dpa}$ and $24 \mathrm{appm} / \mathrm{fpy}$ of helium) and no significant increase of dpa's is achieved by removing the MM.

In order to increase the damage and the helium production, the module should be moved closer to the neutron source, which means, to install it in the place of the CFTM. In this case $12.4 \mathrm{dpa} / \mathrm{fpy}$ and $144 \mathrm{appm} / \mathrm{fpy}$ are expected. The expected ratio He/dpa is, in every cases, higher that the expected value for DEMO.

In terms of tritium generation rate, when the module is installed in the CFTM position, the obtained value fits with the generation rate in the HCLL blanket close to the First Wall. When the module is installed behind CFTM the obtained values are low but representative for the breeder zone of a HCLL blanket.

Concluding, a TRM including an experiment focused on the liquid breeder validation should be installed in the position of the CFTM in order to generate a high amount of tritium (maximum expected in a HCLL blanked) and major damage in the EUROFER capsule walls. In addition, the expected values of damage and tritium generation rate in the TRM are in the range of the expected values for a HCLL DEMO blanket, being installed in any position of the medium flux area of IFMIF.

\subsection{Conceptual design of the experiment}

The present reference design for the Tritium Release Module is shown in the CDR [9] (Fig. 3). The TRM houses eight capsules in which the specimens (disks, pellets, pebbles and specimens for compatibility test) are allocated. Tritium produced in the specimens will be swept by means of helium gas flowing through the capsules and will be carried through pipes to the analyzing equipment. The capsules are cooled by means of another helium jet that flows upwards. The main dimensions of the module have been proposed in [1], providing a square cross-section of $30 \mathrm{~mm} \times 30 \mathrm{~mm}$ and $148 \mathrm{~mm}$ high for each capsule (Fig. 3). 


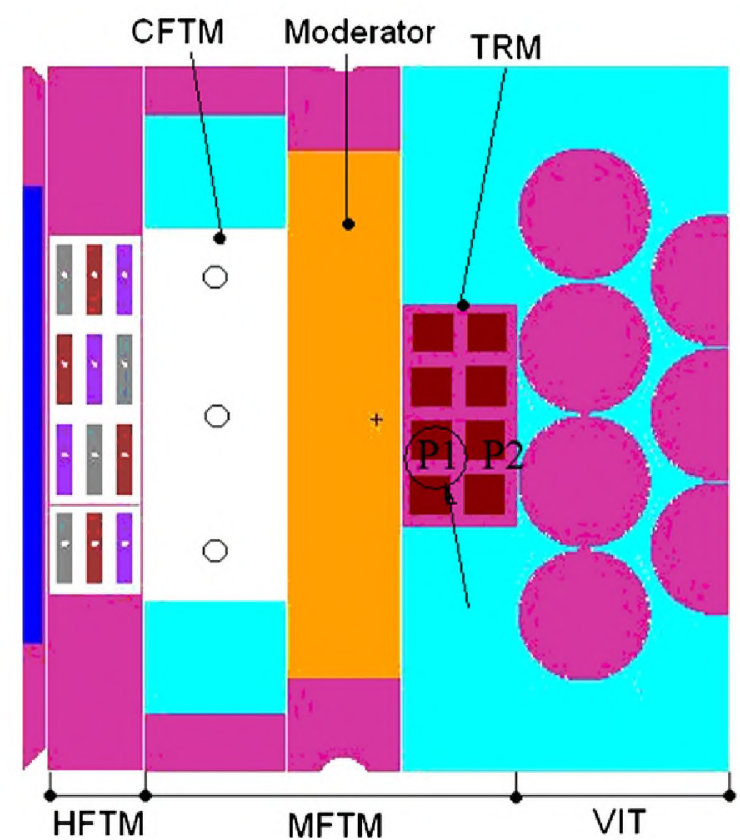

Fig. 3. Tritium Release Module of IFMIF MFTM. Conceptual design [9].

The proposal presented here consist of developing interchangeable rigs to be installed in the current TRM design, introducing minor modification on it, in order to take advantage of the available space and auxiliary systems of this module. Additionally, the design of the experiment is determined by the HCLL blanket LiPb channel conditions between cooling plates. From this starting point, a first conceptual design is shown in Fig. 4 and explained below.

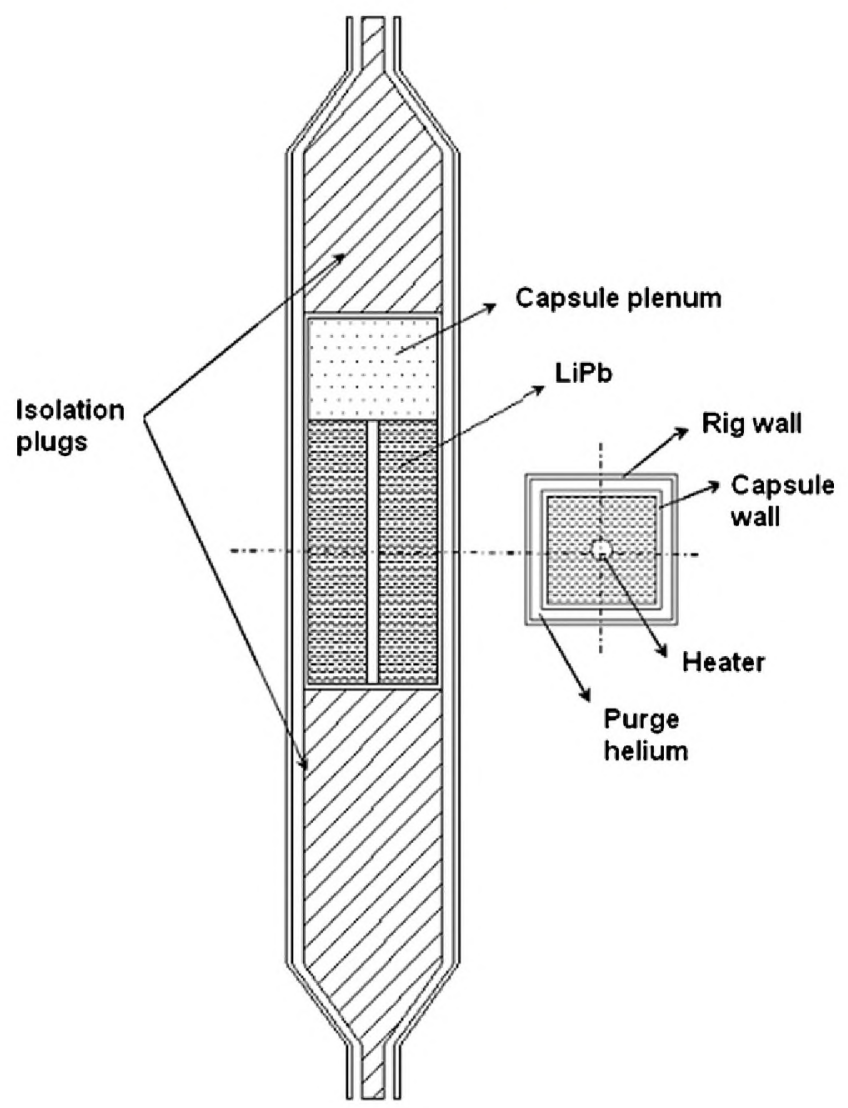

Fig. 4. Rig conceptual design.
The liquid breeder permeation experiment will mainly consist of a rig which contains a closed capsule partially filled with $16 \mathrm{~cm}^{3}$ of LiPb, the surrounding helium purge gas channels and electrical heaters.

The LiPb in the capsule will be in its eutectic composition with $90 \%$ of ${ }^{6} \mathrm{Li}$ enrichment as in the future HCLL blanket [15]. The generated tritium due to the tritium breeding reaction will permeate the capsule walls and will be swept by flowing helium. The conditions of this purge gas can be modified (velocity, composition, etc.) in order to analyze the permeation behaviour. Nevertheless, the experiment will have to be capable of achieving a gas purge flowing at about $40 \mathrm{~m} / \mathrm{s}$ (HCLL helium coolant in cooling plates [3]).

Helium produced in the capsule, assuming that no permeation through the wall takes place, will be released into a $8 \mathrm{~cm}^{3}$ plenum. The volume of the plenum has been designed to widely avoid overpressure in the capsule, considering the maximum expected generated helium in one year of irradiation and maximum temperature of $600^{\circ} \mathrm{C}$ (after one year 4 bar are expected if the volume plenum was $\left.1 \mathrm{~cm}^{3}\right)$.

Taking into account the available space in the present design of TRM, a channel of $2 \mathrm{~mm}$ around the rig has been considered in order to cool the TRM. Therefore, the rig should have $28 \mathrm{~mm} \times 28 \mathrm{~mm}$ of cross-section and $148 \mathrm{~mm}$ high, as maximum. The beam footprint in IFMIF will be $50 \mathrm{~mm}$ high. Consequently, the LiPb sample should take up these $50 \mathrm{~mm}$, the rest of the space being necessary to auxiliary equipment. Also, eight lines of helium purge are available (Fig. 3).

The walls of the LiPb capsule will be made of EUROFER, $1 \mathrm{~mm}$ thick, and will be coated by several fusion relevant anti-permeation coatings materials, e.g., Al-based permeation barriers $\left(\mathrm{Al}_{2} \mathrm{O}_{3}\right.$, for instance), $\mathrm{Er}_{2} \mathrm{O}_{3}$ or TiC, among others [15-17].

Experiments should be carried out with and without coatings in order to compare the measured permeation rates and obtain the anti-permeation capability of each coating. Anti-permeation coatings should be allocated in contact with lithium, in the helium side or both.

In order to achieve the desired sample temperature, an electrical heater (made of a mixture of steel and alumina) is allocated inside the LiPb as shown in Fig. 4. Two isolation plugs are installed on the top and bottom of the sample with triple function: to isolate the rig wall avoiding heat transfer between the sample and the outside wall, to support the capsule, and to distribute the helium purge in the required channel shape. In addition to this, the upper plug will be designed to allow the entrance of cables for the electrical heaters and all the instrumentation required.

Some thermal hydraulic and neutronic calculations have been performed in order to analyze the behaviour of the capsule under operation and to get confidence in the conceptual design of the whole module.

\subsection{Thermal hydraulic calculations}

A three dimensional modelling of the experimental capsule has been performed with the CFX 11 code [18]. A fourth of the geometry (with a structural mesh of 262643 tetrahedral cells) has been simulated by applying symmetry conditions in order to minimize the computational effort.

The main dimensions of the model are $50 \mathrm{~mm}$ high and a square cross-section of $30 \mathrm{~mm} \times 30 \mathrm{~mm}$ [9,1]. Dimensions of the sample, structural material and electrical heaters are shown in Fig. 5. To perform the thermal analysis, the cooling channel and an additional external wall that represents the wall of the container (TRM) which encloses all rigs in the model have been added to the model.

The nuclear heating in the TRM depends on its position: behind the MM or directly behind the HFTM, instead the CFTM. This second option has been suggested in order to increase the damage, 


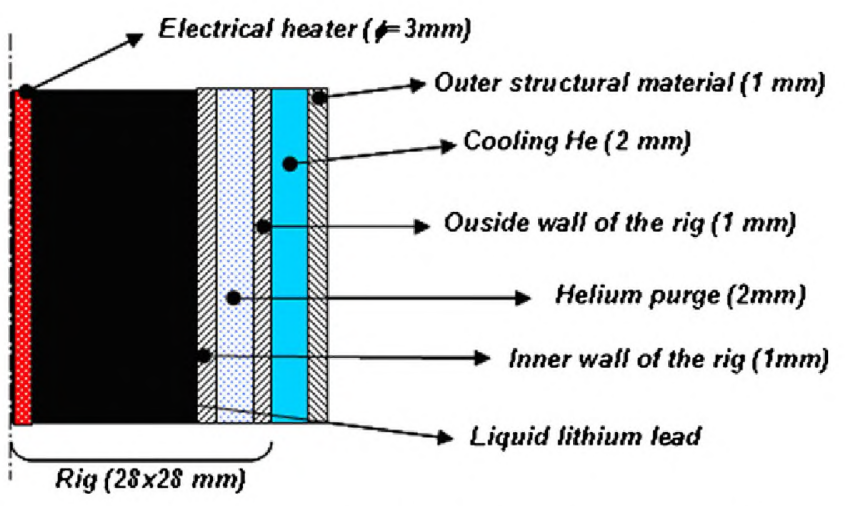

Fig. 5. Schematic view of capsule axial section. Calculation model.

tritium generation rate and gas production in the wall in order to fit the fusion relevant values (see Section 3.1 for details). For that reason it has been considered, for the calculations, as nuclear heating value for the TRM the maximum heating value for TRM, $1 \mathrm{~W} / \mathrm{g}$ [19].

The cooling of the rigs has been designed to release this nuclear heating and to maintain the entire MFTM at low temperature (below $100^{\circ} \mathrm{C}$ ) in order to minimize tritium leaks and assure its mechanical integrity. The electrical heater power and the gas purge temperature were designed to achieve the desired range of temperatures (from $300^{\circ} \mathrm{C}$ to $500^{\circ} \mathrm{C}$ ). The purge helium flows at $40 \mathrm{~m} / \mathrm{s}$ and the pressure was chosen low, $0.3 \mathrm{MPa}$.

The helium coolant flows at $50^{\circ} \mathrm{C}$ and low pressure $(0.3 \mathrm{MPa})$, to avoid excessive mechanical loads to the structures. The helium velocity was chosen high $(200 \mathrm{~m} / \mathrm{s})$ to avoid the laminar regime and the temperature differences along the channel.

For the calculations, natural convection in the LiPb has been considered. In the case of helium purge and helium coolant, natural convection and radiation inside the gaps has been neglected. In addition, $\mathrm{LiPb}$ properties have been chosen temperature dependant [20]. EUROFER, electrical heater material, and helium properties have been were taken from [21,22].

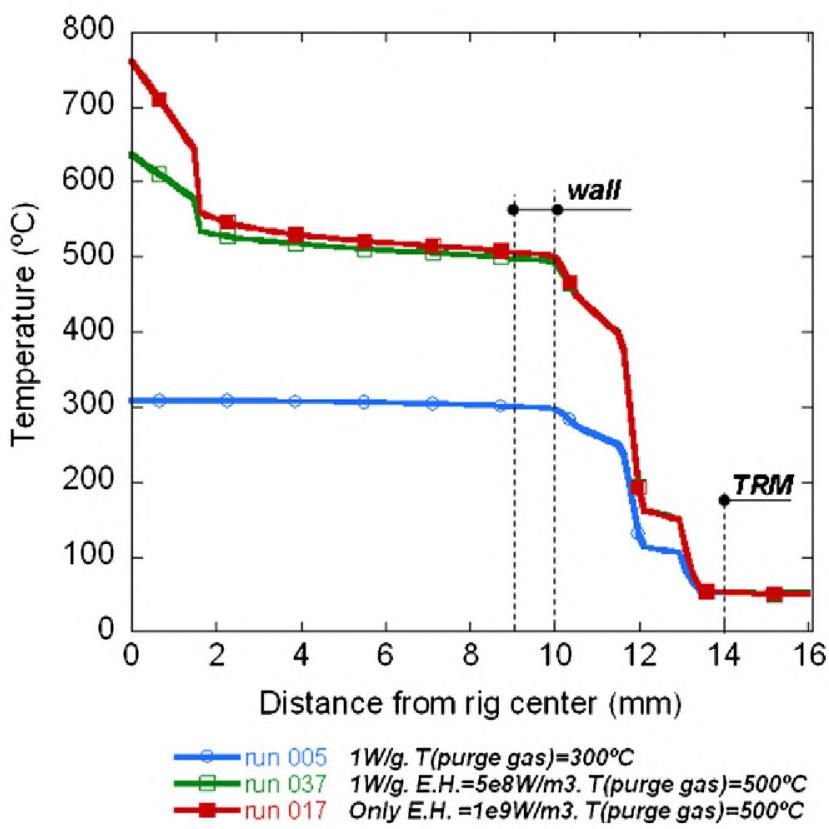

Fig.6. Temperature distribution in the rig cross-section at $25 \mathrm{~mm}$ high of the capsule in three cases: with nuclear heating $(1 \mathrm{~W} / \mathrm{g})$, with both nuclear and electrical heating, and only electrical heating.
Table 2

Expected permeation rates in the three possible positions of the TRM inside the MFTM.

\begin{tabular}{ll}
\hline & Expected permeation rate $\left(\mathrm{at} / \mathrm{m}^{2} \mathrm{~s}\right)$ \\
\hline Position in the MFTM & \\
TRM (without moderator) & $1.1 \mathrm{e} 16$ \\
TRM (with moderator) & $1.25 \mathrm{e} 16$ \\
CFTM & $2.23 \mathrm{e} 16$ \\
\hline
\end{tabular}

The results of thermal analysis (summarized in Fig. 6), show that the desired temperature range is obtained in two extreme situations: with maximum nuclear heating ( $1 \mathrm{~W} / \mathrm{g}$ in CFTM position) and without nuclear heating (beam off situation and maximum sample temperature). The rest of expected cases are comprised thereof. Furthermore, there are no temperature differences in the capsule wall in radial direction and low axial temperature differences (about $10^{\circ} \mathrm{C}$ ). As a result of the nuclear and electrical heating, convective velocities in the order of some $\mathrm{mm} / \mathrm{s}$ have been observed in the lithium lead.

\subsection{Expected measurements}

Permeated tritium together with purge helium will be conducted from the module to the analyzing equipment where the amount of tritium will be measured. Thus, permeation rate for each capsule could be obtained at different temperatures.

The order of magnitude of the permeated rate can be estimated through the tritium concentration balance equation in the capsule. By using a simple approach, the tritium accumulation in the capsule can be defined by Eq. (1), where, in this simplest approach, thermal and velocity $\mathrm{LiPb}$ field have been neglected.

$\frac{\mathrm{d} c}{\mathrm{~d} t}=g-J$

$c$ is the tritium concentration that remain in the capsule, $g$ is the tritium generation rate (obtained by neutronic calculations, see Table 1 ) and $J$ is the permeation rate throughout the capsule wall.

Assuming a diffusion limited permeation model and considering that the tritium partial pressure in helium is very low compared to the partial pressure in LiPb, $J$ can be estimated as follows,

$J=\frac{D_{\mathrm{w}} \mathrm{Ks} \mathrm{s}_{\mathrm{w}} \sqrt{P_{\mathrm{p}}}}{d}$

$D$ is the diffusion of tritium through the wall at the experimental temperature, $\mathrm{Ks}_{\mathrm{w}}$ is the Sievert's constant of tritium in the wall at the experimental temperature, $P_{\mathrm{p}}$ is the tritium partial pressure in the LiPb and $d$ is the wall thickness.

Assuming that Sievert's constant of tritium in $\mathrm{LiPb}, \mathrm{Ks}_{\mathrm{LiPb}}$, is known [4], the partial pressure that determines the permeation rate into the helium channel can be obtained by means of (3).

$c=K s_{\mathrm{LiPb}} \sqrt{P_{\mathrm{p}}}$

Taking into account tritium production rates at MFTM (Table 1) and assuming values shown below for diffusivity and Sievert's constant [4], the expected permeation rates have been represented in Table 2 .

$D_{\mathrm{W}}=1.49 \mathrm{e}-8\left[\mathrm{~m}^{2} \mathrm{~s}^{-1}\right]$ at $480^{\circ} \mathrm{C}$

$\mathrm{Ks}_{\mathrm{W}}=2.27 \mathrm{e}-3\left[\mathrm{~mol} \mathrm{~m}^{-3} \mathrm{~Pa}^{-1 / 2}\right]$ at $48^{\circ} \mathrm{C}$

$\mathrm{Ks}_{\mathrm{LiPb}}=1.02 \mathrm{e}-3\left[\mathrm{~mol} \mathrm{~m}^{-3} \mathrm{~Pa}^{-1 / 2}\right]$ at $540^{\circ} \mathrm{C}$

Values of permeation rate from $1.1 \mathrm{e} 16$ to $2.23 \mathrm{e} 16 \mathrm{at} / \mathrm{m}^{2} \mathrm{~s}$ are expected in the experiment, which can be detected by ionization chambers as tritium detector. From the measured permeation rate can be inferred other important values as the wall permeability $\left(D \times K_{\mathrm{S}}\right)$ under irradiation with and without coating. For 
such purpose, is needed to know the tritium generation rate and capsule temperature. Although the diagnostic strategy of the module is not defined yet, the use of thermocouples and neutron flux detectors in real time (like fission chambers) are foreseen.

\section{Conclusions}

A tritium permeation experiment focused on liquid breeder blanket validation is proposed to be installed in the TRM of IFMIF. The suitability of TRM for such purpose was assessed obtaining values of tritium generation rate, damage and helium production in the expected range of DEMO HCLL blanket values.

A conceptual design of a rig in which the experiment will be allocated has been shown and thermal hydraulic calculations performed in order to demonstrate the suitability of the design for the experiment requirements. The following design issues have been solved:

- The design of the capsule that widely house the amount of generated helium.

- The range of temperatures $\left(300-500^{\circ} \mathrm{C}\right)$ to perform the experiment, covering widely the range of temperatures in the HCLL cooling plates (about $400-500^{\circ} \mathrm{C}$ ) [8]. Furthermore, low temperature gradients have been found in the capsule walls.

- The temperature of the TRM, that is achieved below $100^{\circ} \mathrm{C}$ to reduce tritium leakage and to assure mechanical integrity.

In addition, an estimation of the expected permeation rate was obtained (about $1 \mathrm{e} 16 \mathrm{at} / \mathrm{m}^{2} \mathrm{~s}$ ) in order to know the requirements of the analyzing equipment.

\section{References}

[1] A. Möslang, Design outline of a neutron moderator/reflector with experimental joining verification, EFDA Task TTM0-TTMI-003, 2004.
[2] A Li Puma, Helium-cooled lithium-lead fusion power plant (PPCS model AB) design and integration of in-vessel components and associated systems, EFDA Task TW4-TRP-002-D04, 2005.

[3] A. Li Puma, Helium cooled lithium lead blanket module for DEMO designs and analyses, EFDA Task, TW2-TTBC-001-D01, 2003.

[4] W. Farabolini, Assessment of tritium control in PPCS model AB blanket systems, EFDA Task TW4-TRP-002-D2b, 2005.

[5] J.L. Berton, Helium and tritium leaks in the PPCS VKT-DV cooling circuits, CEA Note Technique, DTN/STPA/LTCG 04/032, 2004.

[6] R. Conrad, LIBRETTO-3: performance of tritium permeation barriers under irradiation at the HFR Petten, Journal of Nuclear Materials 212-215 (1994) 998-1002.

[7] A. Aiello, Determination of hydrogen solubility in lead lithium using sole device, Fusion Engineering and Design 81 (2006) 639-644.

[8] E. Serra, Hydrogen and deuterium permeation measurements on the doublewall tubes material for the water-cooled Pb-17Li DEMO blanket, Fusion Engineering and Design 49-50 (2000) 675-679.

[9] IFMIF International team, IFMIF Comprehensive Design Report, 2004

[10] S.P. Simakov, et al., Advanced Monte Carlo procedure for the IFMIF d-Li neutron source term based on evaluated cross-section data, Journal of Nuclear Materials 307-311 (2002) 1710-1714.

[11] Los Alamos National Laboratory, http://mcnp-green.lanl.gov/about_mcnp5. html, 2004.

[12] F. Wasastjerna, Annals of Nuclear Energy 35 (2008) 438-445.

[13] U. Fischer, Neutronic analyses for the conceptual design of a HCLL reactor, EFDA Task TW4-TRP-002, 2005.

[14] J. Jordanova, Assessment of the shielding efficiency of the HCLL blanket for a DEMO-type fusion reactor, European Atomic Energy Community Under Contract FU06-CT-2004-00025, 2006.

[15] J. Jordanova, Evaluation of nuclear heating, tritium breeding and shielding efficiency of the DEMO HCLL breeder blanket, Fusion Engineering and Design $75-79(2005)$ 963-967.

[16] D. Levchuk, Erbium oxide as a new promising tritium permeation barrier, Journal of Nuclear Materials 367-370 (2007) 1033-1037.

[17] L.V. Boccaccini, Materials and design of the European DEMO blankets, Journal of Nuclear Materials 329-333 (2004) 148-155.

[18] CFX User Guide.

[19] S.P. Simakov, Calculation of complete nuclear response through the entire test cell with consideration of an additional neutron shielding block, EFDA Task TW5-TTMI-003, 2006

[20] E. Mas de les Valls, Lead-lithium eutectic material database for nuclear fusion technology, Journal of Nuclear Materials 376 (2008) 353-357.

[21] S. Gordeev, Optimised design and thermal-hydraulic analysis of the IFMIF/HFTM test section, FZK Report, FZKA 6895, 2003.

[22] F. Tavassoli, DEMO Interim Structural Design Criteria, CEA/DEN/SAC/DMN, 2002 